Editor's Note: These short reviews of a recent paper in the Journal, written exclusively by graduate students or postdoctoral fellows, are intended to mimic the journal clubs that exist in your own departments or institutions. For more information on the format and purpose of the Journal Club, please see http://www.jneurosci.org/misc/ifa_features.shtml.

\title{
Personality Predicts Responsivity of the Brain Reward System
}

\author{
Jan B. Engelmann \\ Department of Psychology, Brown University, Providence, Rhode Island 02912 \\ Review of Beaver et al. (http://www.jneurosci.org/cgi/content/full/26/19/5160)
}

Food is a primary reward. There is no doubt that animals and humans find pleasure in the consumption of food and will work hard to obtain it under certain conditions. Foods differ in their rewarding value, with high-calorie foods typically being highly rewarding. In modern societies, food is readily available and foodrelated advertisements are ubiquitous. Thus, we can be easily motivated to consume rewarding foods even regardless of satiety. The over-consumption of appetizing high-caloric foods has contributed to the dramatic increase in obesity within the past 20 years, making obesity a top 10 global health threat, according to the World Health Organization.

However, not everyone overeats. Individual differences in trait reward sensitivity are associated with increased food cravings, hyperphagia, and body weight (Davis et al., 2004). Reward sensitivity, a personality construct of Gray's biologically motivated Reinforcement Sensitivity Theory is thought to control approach behavior and has been linked to the mesocorticolimbic dopamine system (Pickering and Gray, 1999). Indeed, a network of interconnected brain regions consisting of orbitofrontal cortex, ventral striatum, amygdala, substantia nigra, and ventral tegmental area (Fig. 1) has repeatedly

Received June 5, 2006; revised June 9, 2006; accepted June 9, 2006.

Correspondence should be addressed to Jan B. Engelmann, Department of Psychology, Brown University, 89 Waterman Street, Box 1853, Providence, RI 02912. E-mail: jbe@brown.edu.

DOI:10.1523/JNEUROSCI.2377-06.2006

Copyright $\odot$ 2006 Society for Neuroscience $\quad$ 0270-6474/06/267775-02\$15.00/0 been implicated in mediating approach behaviors related to food reward in animals and humans. A recent study in The Journal of Neuroscience (Beaver et al., 2006) demonstrates a correlation between trait reward sensitivity and neural activity within the reward network.

The authors showed that reward sensitivity, as assessed by the Behavioral Inhibition Scale/Behavioral Activation Scale, predicted neural responses to pictures of appetizing foods in the network of the brain regions outlined in Figure 1. Functional magnetic resonance imaging, in conjunction with a blocked experimental design, was used to record blood oxygenation-level dependent (BOLD) responses while participants passively viewed pictures of foods from four different categories. Volunteers were shown valenced pictures of food, which in separate studies were consistently rated as either highly appetizing and pleasant (appetizing foods) or highly disgusting and nauseating (disgusting foods). The comparison conditions included pictures of bland foods that were consistently rated as less pleasant and disgusting, as well as pictures of nonfood objects. Contrasting BOLD responses during experimental and comparison conditions in the framework of random-effects regression analyses yields activation patterns attributable to the unique characteristics of the experimental stimuli. To obtain activations that were largely attributable to differences in reward value of the food items, the authors compared BOLD responses to pictures of appetizing foods and pictures of bland foods. These contrasts were performed in a select subset of a priori regions of interest within the reward network outlined in Figure 1.

The authors found increased activation in orbitofrontal cortex (appetizing vs nonfood objects) and bilateral ventral striatum (appetizing vs bland foods). Interestingly, there was a dissociation between left and right orbitofrontal cortex such that appetizing food stimuli activated the left orbitofrontal cortex, whereas disgusting food stimuli activated the right orbitofrontal cortex [Beaver et al. (2006), their Fig. 1 (http://www. jneurosci.org/cgi/content/full/26/19/ $5160 / \mathrm{F} 1)]$. These findings suggest that the orbitofrontal cortex is involved in assigning reward value to pictures of food in a valence-dependent manner. The ventral striatum was activated bilaterally in response to appetizing images, whereas the right ventral striatum was activated in response to disgusting images [Beaver et al. (2006), their Fig. 2 (http://www.jneurosci.org/cgi/content/ full/26/19/5160/F2)]. Although the latter finding is somewhat surprising in lieu of a widely accepted view implicating the ventral striatum in reward processes, it is in agreement with the notion that ventral striatal neurons respond not only to rewarding stimuli, but to all salient stimuli, including aversive ones (Becerra et al., 2001). Future studies are required to test contending theories of striatal dopaminergic activity. 


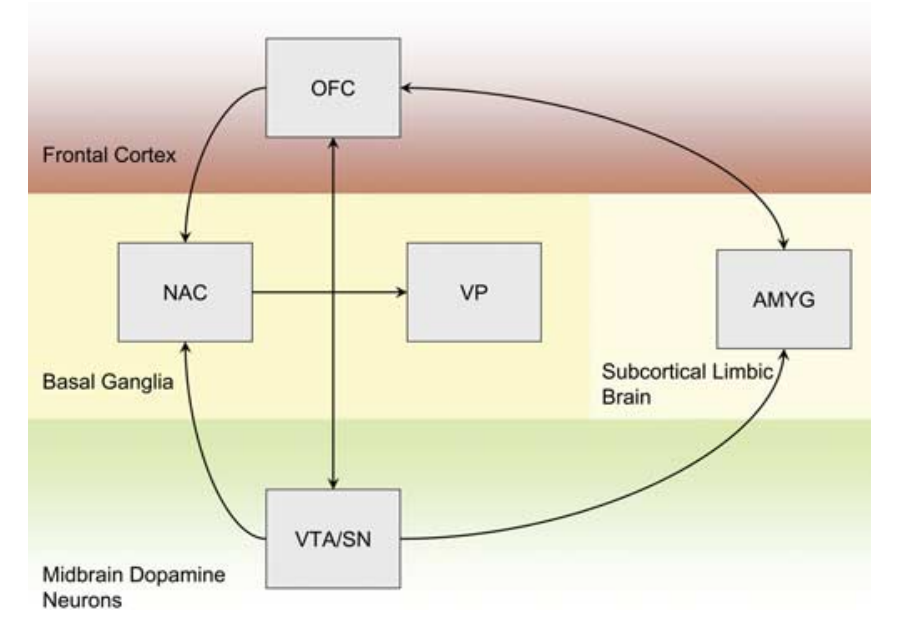

Figure 1. A highly simplified schematic diagram outlines the connections between central nodes in the brain reward system (modified from Berridge and Robinson, 2003). Animal as well as human neuroimaging studies have implicated this network in the hedonic and motivational effects of natural rewards and drugs of abuse, such as food and amphetamine. AMYG, Amygdala; NAC, nucleus accumbens; $0 \mathrm{FC}$, orbitofrontal cortex; VP, ventral pallidum; VTA/SN, ventral tegmental area/substantia nigra.

The authors then used correlation analyses to examine the relationship between trait reward sensitivity and neural responses to images containing appetizing foods. Reward sensitivity correlated significantly with activations resulting from contrasting BOLD responses to appetizing and bland foods [Beaver et al. (2006), their Fig. 3 (http://www.jneurosci.org/ cgi/content/full/26/19/5160/F3)]. These correlations indicate that activity in the reward network in response to viewing appetizing foods increased as a function of increasing trait reward sensitivity. Importantly, this finding was obtained in all a priori regions of interest, underlining the consistency of the effect of trait reward sensitivity on brain activations within the reward network. Furthermore, this pattern of brain activations was observed only after including trait reward sensitivity as a regressor of interest in statistical analyses, stressing the importance of individual differences for future neuroimaging investigations.

In summary, Beaver et al. (2006) provide an important link between human behavioral research that has demonstrated an association between trait reward sensitivity and unhealthy eating habits and animal research implicating the reward network in hyperphagia and increased intake of high-caloric foods. Their findings thus offer a possible explanation for why some individuals overeat compulsively. It will be interesting to establish a more direct link between compulsive overeating and responsivity of the reward system, for instance by correlating activity in the reward system in response to images depicting appetizing foods and body mass index. Finally, an abundance of parallels between obesity and drug addiction points to similarities in the underlying brain mechanisms and neural adaptations that accompany these two conditions (Volkow and Wise, 2005). Future research is needed to identify similarities in the brains of addicted and obese individuals, particularly in the responsivity of the brain reward system to conditioned stimuli predicting food and drugs of abuse, because such cues are important factors in habit formation and mainte- nance. Understanding the brain mechanisms that mediate compulsive overeating can guide our treatment and prevention efforts. Beaver et al. (2006) provide an important step in that direction.

\section{References}

Beaver JD, Lawrence AD, van Ditzhuijzen J, Davis MH, Woods A, Calder AJ (2006) Individual differences in reward drive predict neural responses to images of food. J Neurosci 26:5160-5166.

Becerra L, Breiter HC, Wise R, Gonzalez RG, Borsook D (2001) Reward circuitry activation by noxious thermal stimuli. Neuron 32:927-946.

Berridge KC, Robinson TE (2003) Parsing reward. Trends Neurosci 26:507-513.

Davis C, Strachan S, Berkson M (2004) Sensitivity to reward: implications for overeating and overweight. Appetite 42:131-138.

Pickering AD, Gray JA (1999) The neuroscience of personality. In: Handbook of personality: theory and research, Ed 2 (Pervin LA, John OP, eds), pp 277-299. New York: Guilford.

Volkow ND, Wise RA (2005) How can drug addiction help us understand obesity? Nat Neurosci 8:555-560. 